\title{
A comprehensive study of NGC 2023 with XMM-Newton and Spitzer ${ }^{\star}$
}

\author{
M. A. López-García, ${ }^{1} \dagger$ J. López-Santiago, ${ }^{1}$ J. F. Albacete-Colombo, ${ }^{2}$ \\ P. G. Pérez-González ${ }^{\ddagger} \ddagger$ and E. de Castro ${ }^{1}$ \\ ${ }^{1}$ Departamento de Astrofísica y Ciencias de la Atmósfera, Universidad Complutense de Madrid, E-28040 Madrid, Spain \\ ${ }^{2}$ Centro Universitario Regional Zona Atlántica (CURZA) Universidad Nacional del COMAHUE, Monseñor Esandi y Ayacucho (8500), Viedma (Rio Negro), \\ Argentina
}

Accepted 2012 November 7. Received 2012 November 7; in original form 2011 December 16

\begin{abstract}
Nearby star-forming regions are ideal laboratories to study high-energy emission of different stellar populations, from very massive stars to brown dwarfs. NGC 2023 is a reflection nebula situated to the south of the Flame Nebula (NGC 2024) and at the edge of the $\mathrm{H}$ II region IC 434, which also contains the Horsehead Nebula (Barnard 33). NGC 2023, NGC 2024, Barnard 33 and the surroundings of the O-type supergiant star $\zeta$ Ori constitute the south part of the Orion $\mathrm{B}$ molecular complex. In this work, we present a comprehensive study of X-ray emitters in the region of NGC 2023 and its surroundings. We combine optical and infrared data to determine physical properties (mass, temperature, luminosity and the presence of accretion discs) of the stars detected in an XMM-Newton observation. This study has allowed us to analyse spectral energy distribution of these stars for the first time and determine their evolutionary stage. Properties of the X-ray emitting plasma of these stars are compared to those found in other nearby star-forming regions. The results indicate that the stars that are being formed in this region have characteristics, in terms of physical properties and luminosity function, similar to those found in the Taurus-Auriga molecular complex.
\end{abstract}

Key words: stars: coronae-stars: pre-main-sequence-open clusters and associations: general-X-rays: stars.

\section{INTRODUCTION}

Star-forming regions are the best laboratories to study the different physical processes that give rise to X-ray emission. X-ray photons are little absorbed by interstellar material and young stars show higher levels of X-ray emission than main-sequence stars. High-energy radiation plays an important role in the development and evolution of stellar protoplanetary discs (Bally, O'Dell \& McCaughrean 2000; Scally \& Clarke 2001) and planetary atmospheres (e.g. Penz, Micela \& Lammer 2008) and contributes to the disruption of star formation by causing molecular material blow out. Outflows, jets and winds are the main mechanisms to modify the molecular cloud environment, except for supernova explosions. They displace cloud material but also trigger star formation.

\footnotetext{
^ This publication makes use of data products from the Two Micron All Sky Survey, which is a joint project of the University of Massachusetts and the Infrared Processing and Analysis Center/California Institute of Technology, funded by the National Aeronautics and Space Administration and the National Science Foundation. This research has also made use of the Centre de Données Astronomiques de Strasbourg (CDS) tool ALADIN.

$\dagger$ E-mail: mal@astrax.fis.ucm.es

$\ddagger$ Associate Astronomer at Steward Observatory, The University of Arizona, AZ, USA.
}

The Orion giant molecular complex (see Fig. A1) consists of two main large structures, usually referred to as Orion A and B, and a number of less massive filaments (see Genzel \& Stutzki 1989 for a detailed description). The Orion A molecular cloud contains the Orion Nebula Cluster (ONC) which is the closest OB association to the Sun $(\sim 450 \mathrm{pc})$. Because of its proximity, the ONC is one of the most visited targets for numerous studies, including the relation between cloud kinematics and star formation (see Hartman \& Burkert et al. 2007 and references therein), protostellar and circumstellar disc characteristics (e.g. Fang et al. 2009; Ingleby et al. 2009; Wilson et al. 2011) and the properties of the X-ray emission of young stellar objects and T Tauri stars [see Preibisch et al. 2005 and the rest of the articles of the Chandra Orion Ultradeep Project (COUP) in the special issue of the ApJS]. An extensive study of Orion A combining data from the XMM-Newton and Spitzer missions is just being carried out (see preliminary results in Pillitteri et al. 2010a; Wolk et al. 2010a).

Equally interesting for studies on stellar formation is the Orion $B$ molecular cloud. The complex extends roughly south-north and contains the Horsehead Nebula, NGC 2023, NGC 2024 and several other reflection nebulae, such as NGC 2068 and NGC 2071. The southern part of Orion B (L 1630) bordersthe large H II region IC 434, which is expanding into the molecular cloud. The interface between the molecular cloud and the $\mathrm{H}$ II region (IC 434) is seen as a 
bright ridge of glowing gas with the Horsehead Nebula and several smaller pillars (Mookerjea et al. 2009). The Horsehead Nebula points directly towards the multiple system $\sigma$ Ori, which is ionizing IC 434. At a distance of 350-450 pc, the Horsehead Nebula is the closest pillar to the Sun and represents an ideal laboratory to study the emergence and evolution of X-ray emission in very young stars, their X-ray properties and the influence of X-ray emission and its variability on the heating and evolution of protoplanetary discs (see infrared studies by Bowler et al. 2009; Mookerjea et al. 2009).

NGC 2023 is located about 15 arcmin to the north-east of the pillar. In contrast to NGC 2024, that contains an embedded stellar cluster with more than 200 members and several protostar candidates (Haisch, Lada \& Lada 2000; Skinner, Gagné \& Belzer 2003), NGC 2023 shows low stellar density. Some radio clumps have been detected using the Very Large Array (VLA; Anglada \& Rodríguez 2002; Reipurth et al. 2004). One of them, the source NGC 2023 MM 1, is a confirmed (very cold) class 0 source driving a large and well-collimated molecular outflow (Sandell et al. 1999). Three Herbig-Haro objects were identified by Malin, Ogura \& Walsh (1987): [MOW87] HH1, HH2 and HH3. Mookerjea et al. (2009) associated HH3 with the classical T Tauri star V615 Ori, but the association of $\mathrm{HH} 1$ and $\mathrm{HH} 2$ with a detected source is more uncertain. The authors associated $\mathrm{HH} 2$ with the mid-infrared source MIR-51, while HH1 could be associated with the radio source NGC 2023 MM3, the mid-infrared source MIR-62 or even the near-infrared source NIR-15, merely at 40 arcsec from HH1.

The age of NGC 2023 is not well established. Alcalá et al. (2000) derived ages in the range $0.5<\tau_{\text {age }}<7.0 \mathrm{Myr}$ for stars in the $\mathrm{L}$ 1641 and L 1630 clouds with an X-ray counterpart. For NGC 2024, Eisner \& Carpenter (2003) determined an age of 0.3 Myr from the disc frequency, while Ali et al. (1998) proposed 0.5 Myr as the age of this cluster, based on infrared photometry. The coexistence of both radio clumps and stars without a protoplanetary disc in the region of NGC 2023 (e.g. Mookerjea et al. 2009) suggests that star formation has taken place during several million years, although this hypothesis should be tested robustly.

In this work, we present for the first time a comprehensive study of the X-ray sources in the star-forming region NGC 2023 and its surroundings. We give general X-ray emission characteristics of the sources (temperature, column density and emission measure) and compare them with the results obtained for the nearby regions Taurus (Güdel et al. 2007) and ONC (Preibisch et al. 2005). We complete a multiwavelength study of the X-ray detected sources using data from the literature. In addition, archival observations from the Spitzer infrared observatory are analysed. We then classify the X-ray sources into class I, II and III objects according to their infrared characteristics.

\section{X-RAY OBSERVATION AND DATA REDUCTION}

The XMM-Newton observation of the NGC 2023 reflection nebula (ID 0112640201) was part of an observing program aimed at studying deeply embedded stellar clusters in Orion B. The observation was performed as a single, $30 \mathrm{ks}$ long exposure in 2002 during satellite revolution 419. The European Photon Imaging Camera (EPIC) was used in full-frame mode as primary instrument. The observation was centred at $\alpha=05^{\mathrm{h}} 41^{\mathrm{m}} 47^{\mathrm{s}} .20$ and $\delta=02^{\circ} 16^{\prime} 37^{\prime \prime} .0$. Table 1 summarizes the information of the parameters used for each EPIC detector.

The data reduction was done through the XMM-Newton Science Analysis System (SAS) software, version 10.0. Images and spectra
Table 1. NGC 2023 XMM-Newton observation details on each EPIC detector.

\begin{tabular}{cccc}
\hline & PN & MOS1 & MOS2 \\
\hline Exp. time (ks) & 25.09 & 29.13 & 29.12 \\
Effective exp. time (ks) & 13.60 & 15.00 & 15.80 \\
Filter & Medium & Thin & Medium \\
Obs. mode & Extended full frame & Full frame & Full frame \\
\hline
\end{tabular}

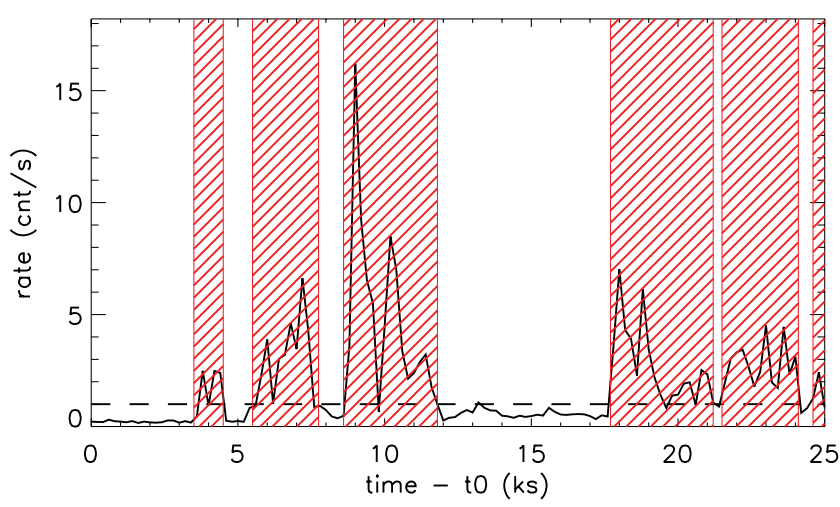

Figure 1. Light curve of the whole EPIC-PN detector at $E>10 \mathrm{keV}$. The dashed line indicates the recommended value for excluding flaring high background by the SAS manual. Shaded regions are those showing high background flaring and, therefore, are those discarded for our analysis.

were produced with the standard SAS tools. We rejected high background periods from the analysis of the light curve of the whole detector (independently for EPIC-MOS and PN) and selected time periods with constant, low X-ray emission at $E>10 \mathrm{keV}$ (Fig. 1). Bad events and noise were also rejected to create the final [good time intervals (GTIs)] event tables. From our analysis, we conclude that the observation was largely contaminated by high X-ray emission background periods (usually attributed to protons and other relativistic particles produced during solar flares). After our processing, the useful exposure time was reduced to $13.6 \mathrm{ks}$ for PN, $15 \mathrm{ks}$ for MOS1 and $15.8 \mathrm{ks}$ for MOS2. Hereafter, we use the cleaned event lists.

In Fig. 2, we show a mosaic of PN and MOS images of NGC 2023 in the energy band $0.3-8.0 \mathrm{keV}$, created with the task emosaic. We used the SAS task edetect_chain to reveal sources at three different energy bands (0.3-1.2, 1.2-2.5 and 2.5-4.5 keV) in PN, MOS 1 and MOS2 separately. A second stage consisted of using the $0.3-8.0 \mathrm{keV}$ energy band to confirm the first detections. A total of 50 sources were detected. After a careful inspection that includes a cross-match with optical and infrared catalogues (see Section 3), we discarded 14 of them because they were multiple or spurious detections, typically at the detector border or close to a bright source. The list of the $\mathrm{X}$-ray sources is given in Appendix A (Table A1). We have removed those sources considered as spurious detections during the optical and infrared analyses (Section 3) from the tables, although we have maintained the original numeration for convenience. X-ray sources of Yamauchi, Kamimura \& Koyama (2000) are identified in the last column of the table as [YKK2000] followed by the ID number given by the authors. 


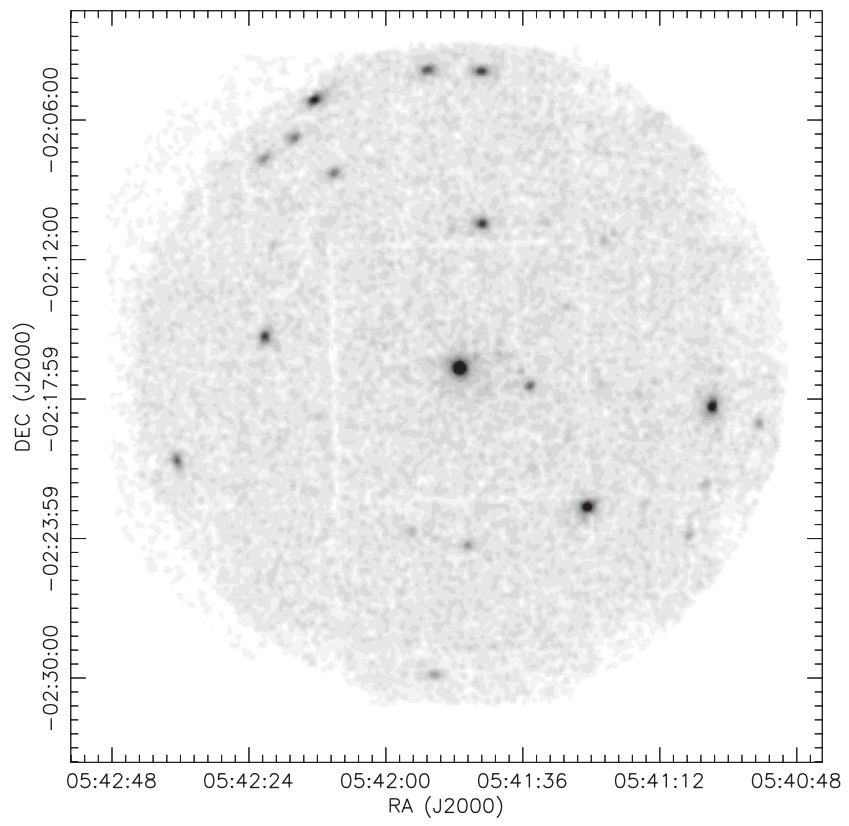

Figure 2. Composite EPIC (MOS+PN) image of the XMM-Newton FoV in the $0.3-8.0 \mathrm{keV}$ energy band.

\section{INFRARED PROPERTIES OF X-RAY SOURCES}

\subsection{Spitzer}

The region of NGC 2023 and its surroundings present a large amount of gas and dust that produces high optical and near-infrared absorption (see Bowler et al. 2009). The absorption by the gas and dust is less dramatic in the mid-infrared, in particular in the Spitzer Infrared Array Camera (IRAC) bands and in radio wavelengths. Mookerjea et al. (2009) used archive Spitzer observations of this region and submillimetric observations performed by themselves with the Submillimetre Common User Bolometric Array (SCUBA; Holland et al. 1999) to create an infrared census of young stellar objects and protostars. Mookerjea et al. (2009) analysed only part of the Spitzer-IRAC observations, close to the Horsehead. The region they selected overlaps only partially with the XMM-Newton observation that is centred on NGC 2023. We have reanalysed the Spitzer data covering the XMM-Newton field of view (FoV) to look for infrared counterparts of all the X-ray detected sources.

For our work, we used the observations with AORKEY numbers 8773120 and 8773632 . Two collections of images are present in those observations for each IRAC channel, corresponding to two different configurations with frame time 0.6 and $10.4 \mathrm{~s}$, respectively. We treated both data sets separately. The regular IRAC pipeline of MOPEX (version 18.4.9) was used for the reduction and the mosaic creation. At the end of the reduction process, we had two mosaics for each IRAC channel and observation, for a total of four mosaics per channel. Photometric analysis was performed in each mosaic independently.

It was not the aim of this work to obtain IRAC fluxes for all the sources in the field. We determined infrared fluxes only for the IR counterparts of the X-ray sources (see Fig. A2). To derive the fluxes, we used the DАОРнот package included in IDL, which is an
Table 2. Aperture correction factors for several different extraction radii.

\begin{tabular}{lcccc}
\hline \multicolumn{5}{c}{ IRAC channel } \\
$\begin{array}{c}\text { Aperture } \\
(\operatorname{arcsec})\end{array}$ & $\begin{array}{c}3.6 \mu \mathrm{m} \\
(\mathrm{mag})\end{array}$ & $\begin{array}{c}4.5 \mu \mathrm{m} \\
(\mathrm{mag})\end{array}$ & $\begin{array}{c}5.4 \mu \mathrm{m} \\
(\mathrm{mag})\end{array}$ & $\begin{array}{c}8.0 \mu \mathrm{m} \\
(\mathrm{mag})\end{array}$ \\
\hline 1.5 & $0.59 \pm 0.04$ & $0.63 \pm 0.04$ & $0.83 \pm 0.04$ & $0.94 \pm 0.06$ \\
2.0 & $0.32 \pm 0.03$ & $0.36 \pm 0.03$ & $0.53 \pm 0.02$ & $0.65 \pm 0.03$ \\
3.0 & $0.13 \pm 0.03$ & $0.14 \pm 0.02$ & $0.22 \pm 0.02$ & $0.36 \pm 0.02$ \\
4.0 & $0.03 \pm 0.02$ & $0.03 \pm 0.02$ & $0.05 \pm 0.02$ & $0.21 \pm 0.03$ \\
\hline
\end{tabular}

adaptation of the FORTRAN code also used in IRAF ${ }^{1}$. Aperture photometry with three different extraction radii $(2,3$ and 4 arcsec) was performed. For the background (sky) subtraction, we used an annulus with inner and outer radii 20 and 30 arcsec, respectively. The mosaics performed by MOPEX are in units of $\mathrm{MJy} \mathrm{s}^{-1}$. We transformed them to mJy and applied an aperture correction. Aperture corrections were estimated from semi-empirical point response functions $(\mathrm{PRFs})^{2}$ given in the Spitzer Manual. Their values are given in Table 2 for each channel. The uncertainties include the effects of typical World Coordinate System (WCS) random alignment errors (always less than 1 pixel; see Pérez-González et al. 2008). For each source, we determined magnitudes in AB system and Vega system. The latter were determined using the flux to magnitude conversion of the Galactic Legacy Infrared Mid-Plane Survey Extraordinaire (GLIMPSE) $^{3}$. For each infrared source, fluxes determined with extraction radii of 2, 3 and 4 arcsec are very similar except for the two most brilliant IRAC sources that show differences of up to 10 per cent in the four channels. The same statement is applicable to the comparison between observations with frame times 0.6 and $12 \mathrm{~s}$. In the latter case, the differences arise from the fact that those sources are very close to the saturation regime for long exposures. To avoid problems with those sources, we decided to use only the images with shorter exposure times. The Spitzer photometry for our sources is given in Table A3. We note that Src 24 was not crossidentified with a unique IRAC counterpart because of the proximity of several infrared sources that contaminate the photometric measures. In the tables, we give photometry of the closest star to this Src 24 for completeness. However, those values are not reliable and we did not use them in our study.

Fig. 3 shows a colour-colour diagram of the four IRAC channels for those of our sources with a counterpart in the four bands. We have marked the typical location of infrared class I, II and III objects and the loci of broad- and narrow-line active galactic nuclei (AGNs) as shown by Stern et al. (2005). Source ID numbers for class I and II objects are overplotted. The cross at the right bottom corner represents the mean error bar in both colours. The typical error in the colours of bright sources is $0.1 \mathrm{mag}$. Faint sources show error bars with values as large as $1.2 \mathrm{mag}$ in some cases.

The main concentration of sources is detected upon class III that are usually identified with weak-line $\mathrm{T}$ Tauri and main-sequence stars (asterisks in Fig. 3). Five sources are situated inside the boundaries of the class II type objects and another two are very close to those boundaries. Stellar sources with infrared class II objects' nature are typically identified with classical T Tauri stars. In Fig. 3, we have plotted them as filled circles. Finally, two sources have colours

\footnotetext{
${ }^{1}$ http://iraf.noao.edu/

2 'Spitzer pixels are relatively big relative to point sources, SO MOPEX fits with PRFs, which take into account intra-pixel sensitivities.'

${ }^{3}$ http://www.astro.wisc.edu/sirtf/
} 


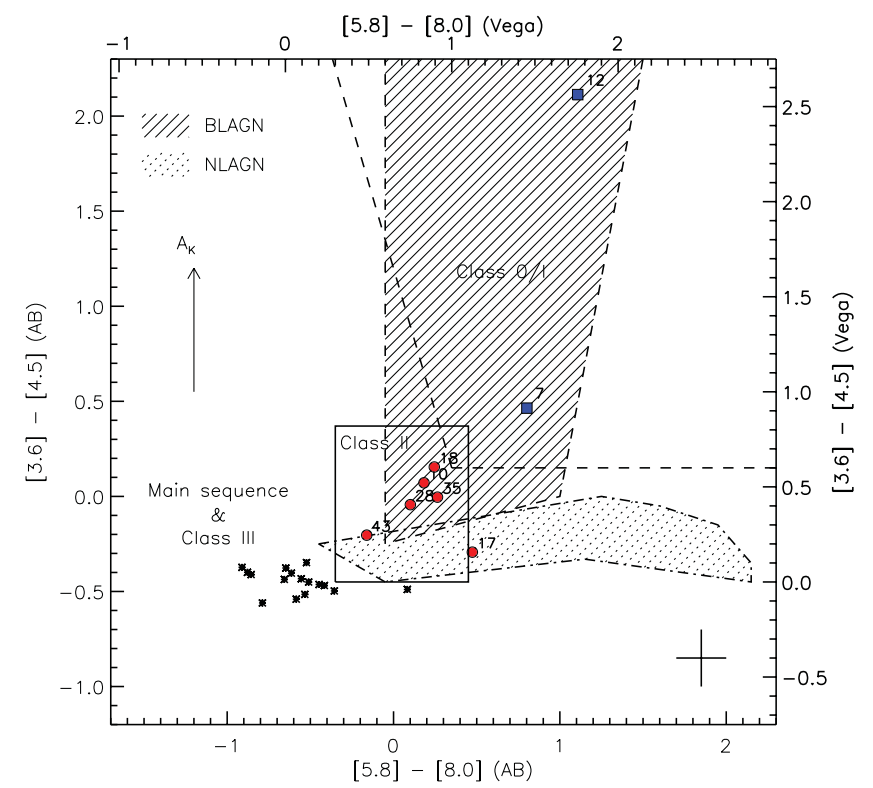

Figure 3. IRAC colour-colour diagram of the X-ray sources of NGC 2023 and its surroundings. The locus of broad- and narrow-line AGNs is marked. Extinction vector is also overplotted. The cross symbol at the left bottom corner indicates the typical error for sources in our sample. Squares are sources classified as class I objects. Dots are class II objects while asterisks are stars classified as main-sequence or class III objects. The extinction vector is $A_{\mathrm{K}}=5$ mag.

typical of class I objects (squares in the figure). Stellar sources with such infrared properties use to be related to protostars and T Tauri stars with circumstellar envelopes. A note of caution must be given here. Very absorbed class II objects may present IRAC colours typical of class I objects. Therefore, the two sources in Fig. 3 classified as class I objects may be absorbed class II stars. In fact, one of the sources has been previously classified as a class II star in the literature. This is the case of the object detected by Mookerjea et al. (2009) with Spitzer and SCUBA (named VLA 3 and MIR 46 in their paper, source number 7 in our work, Src 7).

\subsection{Other photometric data from the literature}

We complemented our study by cross-correlating our sample against different catalogues. In particular, we used the second version of The Amateur Sky Survey (TASS) Mark IV photometric catalogue (Droege et al. 2006; Droege, Richmond \& Sallman 2007) for $V$ magnitudes, DENIS data base third release (DENIS Consortium 2005) for I magnitudes, the Two Micron All Sky Survey (2MASS) data base (Skrutskie et al. 2006) for near-infrared and the Wide-field Infrared Survey Explorer (WISE) preliminary data release (Cutri et al. 2012) for mid-infrared. The cross-correlation was performed through the specific ALADIN Java tool (Bonnarel et al. 2000). We used a search radius of 5 arcsec to prevent the loss of coincidences due to the XMM-Newton positional uncertainties. We finally crossmatched our sample with the list of stars of Mookerjea et al. (2009).

Tables A2 and A3 summarize the photometric data for each one of our sources. We note that 13 out of the 50 sources detected in $\mathrm{X}$-rays have no counterpart in any of the catalogues mentioned above. We considered them as spurious X-ray detections and, therefore, we removed them from the tables. We maintained Src 23 because it is situated at 11 arcsec from an optical source. We cannot discard this source as a real detection. The remaining sources have a counterpart in DENIS and/or 2MASS, except for source 7 (VLA

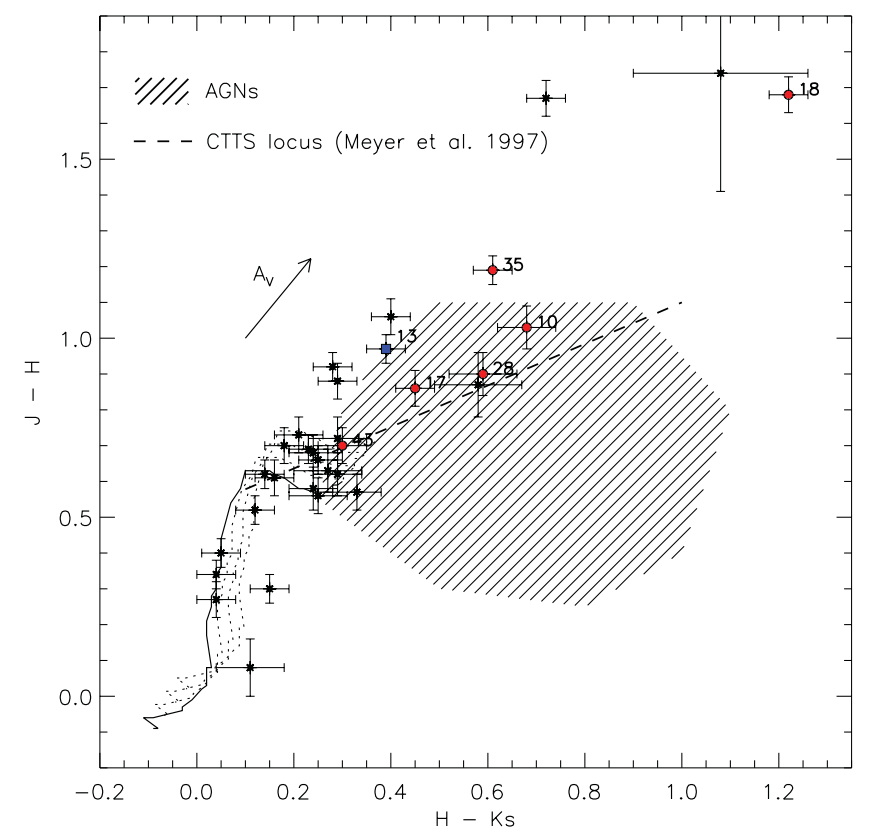

Figure 4. 2MASS colour-colour diagram of the X-ray sources of NGC 2023 and its surroundings. The dwarf stars' main sequence is plotted as a continuous line. The extinction vector is $A_{\mathrm{V}}=3 \mathrm{mag}$. The dotted lines correspond to the dwarfs' main sequence with $A_{\mathrm{V}}=0.5,1.0$ and 1.5 mag. Symbols are the same as in Fig. 3.

3 in Mookerjea et al. 2009, see Section 3.1) and Src 12 (only Spitzer photometry). In Fig. 4, we plot the 2MASS colour-colour diagram for these sources. The main sequence for unabsorbed (dwarf) stars is overplotted as a continuous line. The shaded region in the figure is the locus of the AGNs determined by us using data from Kouzuma \& Yamaoka (2010). The position of most of the stars in the colour-colour diagram indicates the high interstellar extinction in this star-forming region. As shown in Fig. 3, we identified class II objects with its $\mathrm{X}$-ray source ID number.

We estimated the value of the extinction $\left(A_{\mathrm{V}}\right)$ for each star, from the 2MASS colour-colour diagram (Fig. 4). Our results are given in Table A2. These will be discussed and compared with those obtained from spectral energy distribution (SED) fitting in Section 3.4.

Barrado et al. (2011) showed that the $I-J$ versus $J-[3.6]$ colour-colour diagram can be used to reject extragalactic objects from X-ray selected samples, as AGNs are well separated from stars. Fig. 5 shows the same diagram for our 32 sources with a DENIS counterpart. Src 10 and Src 28 are situated close to the boundaries of extragalactic sources, but they have been clearly identified with stars in the optical images (in fact, Src 10 is V615 Ori). Only Src 18 and Src 35 may be absorbed quasars, according to the direction of the extinction vector (in Tables A2 and A3 they are identified as possible AGNs).

\subsection{Age of the NGC 2023 members}

It was mentioned in Section 1 that the age of the star-forming region around NGC 2023 is not well known. There are very few works about this issue in the literature (see Meyer et al. 2008 for a review). Alcalá et al. (2000) gave a range of ages for the stars belonging to the Orion Molecular Complex, but there is no particular study on each individual region. Ages determined for these stars are in the range $0.5-7.0 \mathrm{Myr}$. 


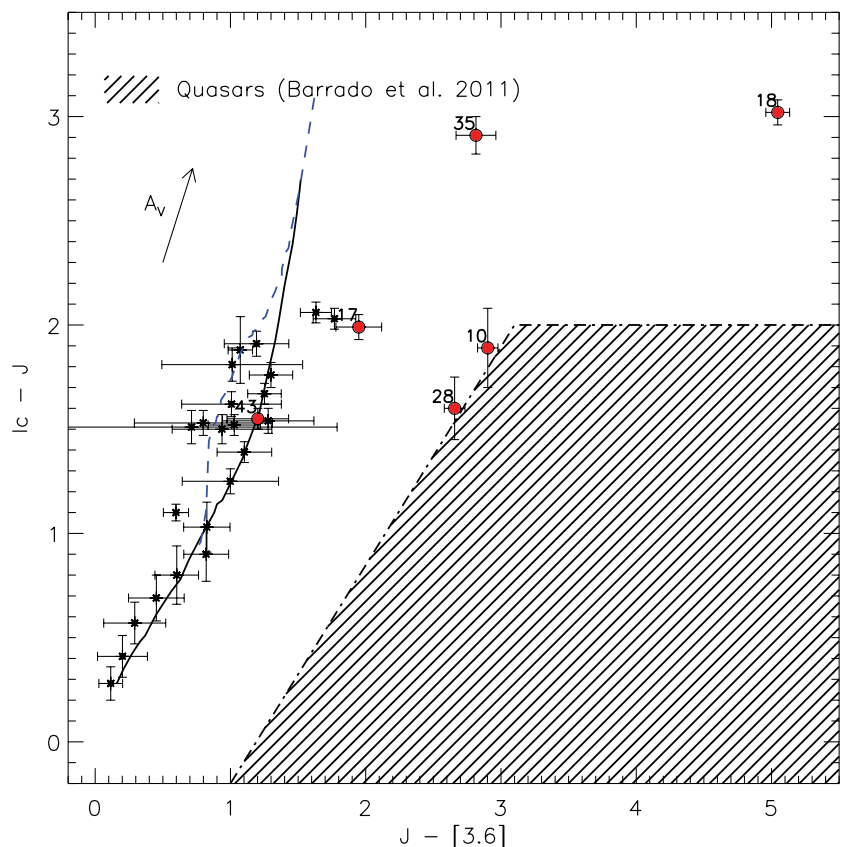

Figure 5. $I-J$ versus $J-[3.6]$ colour-colour diagram of the X-ray sources of NGC 2023. The continuous line represents the ZAMS and the dashed line is the $1 \mathrm{Myr}$ isochrone (Baraffe et al. 1998). The shaded region is the locus of quasars (adapted from Barrado et al. 2011). Symbols are the same as in Fig. 3. The extinction vector is $A_{\mathrm{V}}=1.5 \mathrm{mag}$.

In Fig. 6, we show evolutionary tracks from Baraffe et al. (1998), with an extension to stellar masses over $1.6 \mathrm{M}_{\odot}$ from Siess et al. (2000), in a 2MASS colour-magnitude diagram. We assumed a mean distance of $400 \mathrm{pc}$ for the region. The isochrones are 1, 3 and $7 \mathrm{Myr}$ and the zero-age main sequence (ZAMS). We also plot

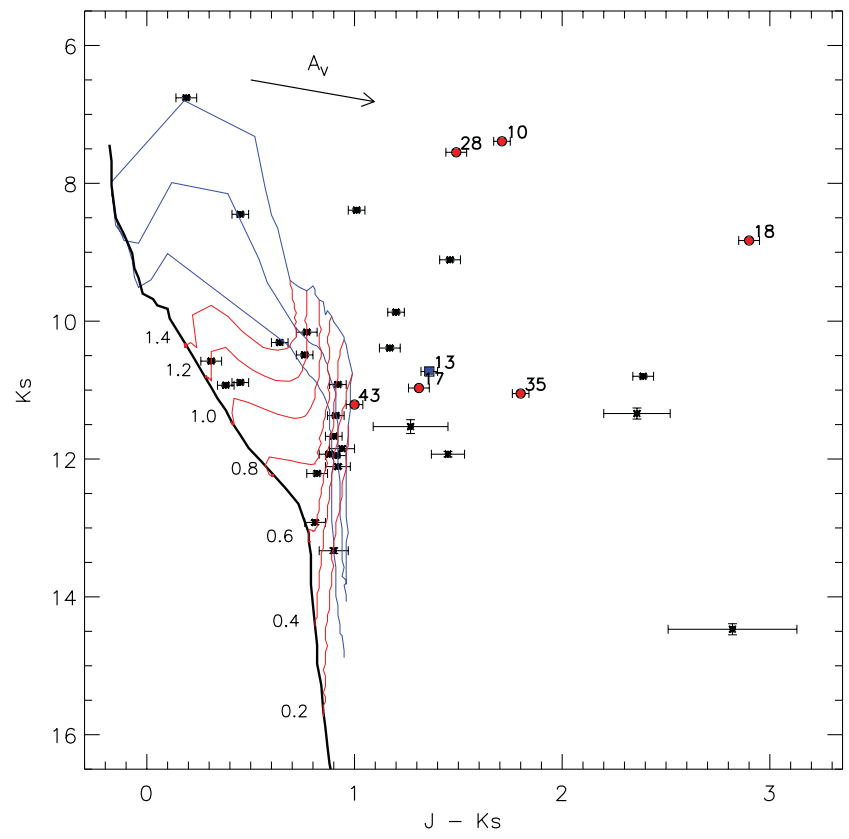

Figure 6. $K_{\mathrm{S}}$ versus $J-K_{\mathrm{S}}$ colour-magnitude diagram of the X-ray sources of NGC 2023. The lines are evolutionary tracks from Baraffe et al. (1998) and Siess, Dufour \& Forestini (2000), with the ZAMS, 7, 3 and $1 \mathrm{Myr}$ isochrones and several mass tracks $(d=400 \mathrm{pc})$. Symbols are the same as in Fig. 3. The extinction vector is $A_{\mathrm{V}}=5 \mathrm{mag}$. tracks for masses ranging from 0.2 to $1.4 \mathrm{M}_{\odot}$. Although there is a concentration of low-mass stars around the $7 \mathrm{Myr}$ isochrone, the density of isochrones in that region of the diagram makes difficult to assure that this is the age for the cluster. In fact, the most massive stars seem to follow younger isochrones. Therefore, we may only affirm that the age of the stars in NGC 2023 is in the approximate range 1-7 Myr.

\subsection{SED fitting}

To investigate in more detail the nature of our X-ray sources, we performed SED fitting using the photometric data we compiled for each source (Tables A2 and A3). We took full advantage of the grid of young stellar object radiation transfer models of Robitaille et al. (2007). In the model, we permitted the extinction to vary only from zero to the maximum value given by the 2MASS colour-colour diagram (Fig. 4). Extinction and distance are the only parameters external to the model.

Fitting was performed for the 32 sources in our sample with a DENIS counterpart, to include at least one optical band. This should improve spectral-type determination. The blue spectrum of a class II object is usually affected by accretion, as it produces an excess in the blue and ultraviolet spectral range. In Figs A3 and A4, we show the best fit for those 32 sources. In some cases, there is more than one model that fits well our data. For those cases, we overplot them as grey lines. We have assumed as good models those in which $\left(\chi^{2}-\chi_{\text {best }}^{2}\right) / \chi_{\text {best }}^{2} \leq 0.1$. In most of those cases, it is not possible to discard any of the fits. We would need some point in the submillimetre spectral range (see discussion in Section 5).

The classification into the different infrared classes given in Tables A2 and A3 was performed according to the results of the SED fitting, using the parameter $\alpha$, defined as the slope of the SED beyond $2.2 \mu \mathrm{m}$ (see Robitaille et al. 2007). We used every point of the SED for this purpose, joining WISE and Spitzer data. A review on the classification method can be found in Stahler \& Palla (2005). We classified as class III objects those sources for which an absorbed stellar model is enough to fit the observed SED $(\alpha \leq-1.5)$. Only Src 14 may show a very thin circumstellar disc (see Fig. A3). Nevertheless, the data fits well also to a stellar photosphere. Class II objects $(-1.5<\alpha \leq 0.0)$ show an obvious excess at the IRAC bands (see Fig. A4). For those sources, any attempt of fitting a stellar model without disc was unsuccessful. Src 13 and Src 17 show characteristics of both class I and II objects. Their SED first decreases between 1.0 and $5.4 \mu \mathrm{m}$, but then increases again. We have classified them as class I/II objects. Finally, there are several sources that have SEDs typical of class III objects but with an excess at wavelengths longer than $10 \mu \mathrm{m}$. We have classified them as class II/III objects. Src 42 is situated in the IRAC colour-colour diagram (Fig. 3) in a position that is usually occupied by stars with transition discs. Our classification into infrared classes using the SED coincides very well with that made first using the IRAC colour-colour diagram, except for Src 44, the SED of which shows an excess above $10 \mu \mathrm{m}$ but that in the colour-colour diagram is situated inside the boundaries of class III objects.

Finally, we note that the SED fitting tool of Robitaille et al. (2007) gives an age for each star. This value is determined through isochrone fitting, so its result depends strongly on the model chosen. Robitaille et al. (2007) use the Siess et al. (2000) pre-mainsequence evolutionary models that differ slightly from the Baraffe et al. (1998) models. The tool also tends to fit low-age isochrones even when a simple photosphere model is suitable for the observed 
SED. Therefore, the results of the fitting tool should be used with some caution.

Ages determined using the Robitaille et al. (2007) fitting tool are in the range 5-9 Myr for most of the stars, what may agree with the results presented in Section 3.3. Nevertheless, for stars showing larger infrared excesses, ages are in the range 0.5-2 Myr.

\subsection{Extinction, stellar masses, temperatures and luminosities}

Table A4 summarizes the results of the SED fitting for the 32 sources with an optical counterpart (see Section 3.4). For sources with more than one good fit, we give only parameters for the best fit. For class II objects, the accretion rate and evolutionary stage are also given. To account for the evolutionary stage of young stellar objects, Robitaille et al. (2007) defined a parameter based on physical properties of the circumstellar envelopes, such as mass and accretion rate from it. The authors distinguished between stars with significant infalling envelopes (Stage 0/I objects), stars with optically thick discs and thin envelopes (Stage II objects) and stars with optically thin discs (Stage III objects). We have determined this stage for our sources and included it in Table A4. We observe that this classification coincides very well with that given by the IRAC colour-colour diagram or the slope of the SED. Class I objects present properties of Stage I objects, while class II objects are classified as Stage III objects following this method.

We previously mentioned that parameters listed in Table A4 are those given by the model. In fact, the extinction $\left(A_{\mathrm{V}}\right)$ and the distance are parameters external to the radiative model (see Robitaille et al. 2007). In addition, stellar mass and radius are determined from evolutionary tracks (Robitaille 2008). In essence, the parameters derived from the model are the temperature and luminosity of the star.

With respect to the interstellar extinction, the values coming from the SED fitting tool are quite similar to those determined with the 2MASS colour-colour diagram if one takes error bars into account for stars showing low extinction. For the remaining stars, there is a trend of $A_{\mathrm{V}}$ determined using the colour-colour diagram to be higher than that coming from the SED fitting tool. The median of the ratio between $A_{\mathrm{V}}$ determined with both methods is 1.35 , or 1.5 if only stars with $A_{\mathrm{V}} \geq 1$ are considered. These stars are mainly those for which the extinction determined with both methods does not coincide even when error bars are taken into account.

\section{X-RAY SPECTRAL ANALYSIS}

In Section 2, we showed that the X-ray observation of the NGC 2023 nebula was affected by high background periods (see Fig. 1). The highly variable background prevented us to perform a study about variability of the detected sources. Therefore, our study was limited to the spectral analysis. The X-ray spectrum of sources with more than approximately 100 net counts was compared (using fitting procedures) to hot plasma models using XSPEC (Arnaud et al. 1996, 2004). The software first loads the data previously extracted using specific SAS tools (source and background spectra, response matrix and ancillary file) and then generates the plasma model. Two hot (diffuse) plasma models are available in XSPEC: mekal (Mewe et al. 1985, 1986; Kaastra et al. 1992; Liedahl, Osterheld \& Goldstein 1995) and apec (Smith et al. 2001a). To be consistent with our previous works (e.g. López-Santiago \& Caballero 2008; LópezSantiago, Albacete Colombo \& López-García 2010a), we chose the latter for the present analysis. The apec uses the atomic data contained in the Astrophysical Plasma Emission Database (APED; Smith et al. 2001b).

Due to the limitations of our observation in terms of counts because of the time lost due to the high background periods - we used only $1 T$ or $2 T$ models for the fits. The results of the spectral fitting are given in Table 3 for those stars with accurate fits. For several stars, the value of the abundance obtained during the fit was unconstrained. In those cases, we fixed the abundance to $Z / Z_{\odot}=$ 0.2. Although some authors have used other values in their works (e.g. Stelzer, Micela \& Neuhäuser 2004; Giardino et al. 2007a; Robrade \& Schmitt 2007), in other stars of this star-forming region we always find a value of $Z / Z_{\odot}$ very close to 0.2 . We used $\chi^{2}$ statistics to obtain the goodness of our fits. Energy channels were then grouped to contain at least 15 counts in each energy bin.

\section{DISCUSSION}

Spectral fitting was performed for the 11 sources in our sample with at least 500 counts (combining PN and MOS detectors). The results are shown in Table 3.

Table 3. Results of the X-ray spectral fitting to hot plasma models for stars in NGC 2023. Errors are at 90 per cent confidence level.

\begin{tabular}{lcccccccc}
\hline ID & $\begin{array}{c}N_{\mathrm{H}} \\
\left(\times 10^{22} \mathrm{~cm}^{-2}\right)\end{array}$ & $\begin{array}{c}k T_{1} \\
(\mathrm{keV})\end{array}$ & $\begin{array}{c}k T_{2} \\
(\mathrm{keV})\end{array}$ & $\mathrm{EM}_{1} / \mathrm{EM}_{2}$ & $\begin{array}{c}Z \\
\left(\mathrm{Z}_{\odot}\right)\end{array}$ & $\begin{array}{c}\chi^{2} \\
(\mathrm{dof})\end{array}$ & $\begin{array}{c}f_{\mathrm{X}}{ }^{a} \\
\left(\times 10^{-13} \mathrm{erg} \mathrm{cm}^{-2} \mathrm{~s}^{-1}\right)\end{array}$ & $\begin{array}{c}\log L_{\mathrm{X}}{ }^{b} \\
\left(\mathrm{erg} \mathrm{s}^{-1}\right)\end{array}$ \\
\hline 3 & $0.00_{-0.00}^{+0.01}$ & $0.62_{-0.11}^{+0.09}$ & $1.26_{-0.13}^{+0.21}$ & 0.70 & $0.23_{-0.05}^{+0.05}$ & $1.29(276)$ & 3.95 & 29.2 \\
10 & $0.50_{-0.04}^{+0.05}$ & $2.49_{-0.52}^{+0.34}$ & - & - & $0.33_{-0.16}^{+0.22}$ & $1.29(140)$ & 12.20 & 31.3 \\
17 & $0.22_{-0.08}^{+0.44}$ & $1.24_{-0.53}^{+1.62}$ & - & - & $=0.2$ & $1.27(54)$ & 0.57 & 30.3 \\
20 & $0.07_{-0.05}^{+0.05}$ & $0.78_{-0.80}^{+0.16}$ & $1.64_{-2.32}^{+2.32}$ & 2.59 & $0.13_{-0.03}^{+0.13}$ & $0.88(78)$ & 2.30 & 30.5 \\
22 & $0.05_{-0.04}^{+0.06}$ & $0.34_{-0.04}^{+0.07}$ & $1.21_{-0.20}^{+0.17}$ & 1.29 & $0.18_{-0.06}^{+0.10}$ & $0.83(120)$ & 3.30 & 30.5 \\
27 & $0.41_{-0.21}^{+0.33}$ & $18.3_{-13.7}^{+60.0}$ & - & - & $=0.2$ & $0.51(10)$ & 1.38 & 30.0 \\
28 & $0.44_{-0.05}^{+0.07}$ & $0.28_{-0.04}^{+0.05}$ & $1.24_{-0.05}^{+0.04}$ & 1.02 & $0.16_{-0.03}^{+0.03}$ & $1.10(602)$ & 33.41 & 31.9 \\
34 & $0.40_{-0.08}^{+0.10}$ & $2.73_{-0.58}^{+1.08}$ & - & - & $=0.2$ & $1.26(59)$ & 6.99 & 30.9 \\
36 & $0.24_{-0.10}^{+0.16}$ & $2.14_{-0.81}^{+1.76}$ & - & - & $=0.2$ & $0.72(61)$ & 2.17 & 30.4 \\
41 & $0.08_{-0.02}^{+0.03}$ & $2.36_{-0.61}^{+0.55}$ & - & - & $0.15_{-0.10}^{+0.14}$ & $1.18(271)$ & 11.53 & 30.6 \\
50 & $0.22_{-0.08}^{+0.08}$ & $2.00_{-0.62}^{+1.86}$ & - & - & $=0.2$ & $1.33(65)$ & 3.41 & 30.2 \\
\hline
\end{tabular}

${ }^{a}$ Unabsorbed flux in the energy band [0.3-8.0] keV. ${ }^{b}$ Unabsorbed X-ray luminosity in the energy band [0.3-8.0] keV. We assumed $d=450 \mathrm{pc}$ (the distance to the ONC) for all the sources. 
The position in the colour-colour diagram of Fig. 5 of the 11 spectroscopically sources studied in this work indicates they are indeed stars. According to that diagram, only Src 18 and Src 35 may be (absorbed) extragalactic sources. General X-ray parameters obtained for these 11 stars are very similar to those observed in stars of the same class in other star-forming regions (e.g. Getman et al. 2005; Güdel et al. 2007). Among them, three are classified as class II from their SEDs (see Tables A2 and A3). They are Src 10 (V615 Ori), Src 17 and Src 28. Another two stars likely have transition discs (Src 36 and Src 41). The remainder are classified as class III or main-sequence stars.

Coronal temperatures are consistent with being young $\mathrm{T}$ Tauri stars (e.g. Güdel et al. 2007). Only Src 27 shows an extremely high temperature, although it is unconstrained (this is the source with the lowest number of counts we could fit). Unabsorbed X-ray fluxes in the energy band [0.3-8.0] keV were determined for the 11 sources for which a spectral fitting was performed and then transformed to luminosities using the distances obtained during the SED fitting procedure (Table A4). We note that for HD 37805 (Src 3), the distance given by the SED fitting tool is slightly underestimated (Hipparcos distance for HD 37805 is $d=82 \pm 7 \mathrm{pc}$ ). However, this is the only star for which we could find a value for its distance in the literature. Therefore, an interpolation of this result to the remaining stars should not be done. In fact, the distance obtained with this procedure for V615 Ori ( $\operatorname{Src} 10, d=371 \mathrm{pc}$ ) is consistent with some results found in the literature: there are hints that the Horsehead Nebula is a few parsecs closer to us than $\sigma$ Orionis that is situated at approximately 385 pc (see Caballero \& Solano 2008 and references therein). V615 Ori must be closer than the Horsehead Nebula as it is observed at the base of the nebula, in front of it. Therefore, the use of the distances obtained from the SED fitting tool seems to be a good option, undoubtedly better than using the same distance for all the sources. Observed X-ray luminosities are indicative of the shallowness of this observation. Typical X-ray luminosities of young $\mathrm{M}$ dwarfs are in the range $L_{\mathrm{X}} \sim 10^{28.5}-10^{30} \mathrm{erg} \mathrm{s}^{-1}$ (e.g. Preibisch \& Feigelson 2005). In this observation, the completeness limit is $f_{\mathrm{X}} \sim 5 \times 10^{-14} \mathrm{erg} \mathrm{cm}^{-2} \mathrm{~s}^{-1}$, what corresponds to $L_{\mathrm{X}} \sim$ $10^{30} \mathrm{erg} \mathrm{s}^{-1}$ at a distance of $400 \mathrm{pc}$. Stelzer et al. (2012) pointed out that there is a trend of a mass dependence of the X-ray emission level throughout the various evolutionary stages of young stellar objects. Comparing the luminosities of class II and III objects in our sample, we do not find such differences, but our sample is too small to achieve robust results.

With the aim of comparing general results of our X-ray analysis of NGC 2023 with other star-forming regions, we plotted the $\mathrm{X}$-ray luminosity cumulative distribution function of our sample, the ONC and the Taurus Molecular Complex together (see Fig. 7). The data of those regions were taken from Getman et al. (2005) and Güdel et al. (2007), respectively. For each star-forming region, we plot the whole sample accessible in those works with no distinction between infrared classes. For NGC 2023, we discarded HD 37805 (Src 3), that is likely a field star, and those stars for which we did not fitted a model to their SEDs (Src 7, Src 12, Src 16 and Src 24). For the stars for which a spectral analysis with XSPEC could not be performed, we determined X-ray fluxes from the observed count rate in the following way. We used the subsample of 11 stars in Table 3 for which X-ray spectral fitting was done to determine a conversion factor between observed count rate and (absorbed) flux. This conversion factor was determined for each star and each EPIC detector (PN, MOS1 and MOS2) independently. Then, we performed a linear regression to each one of the three data sets.

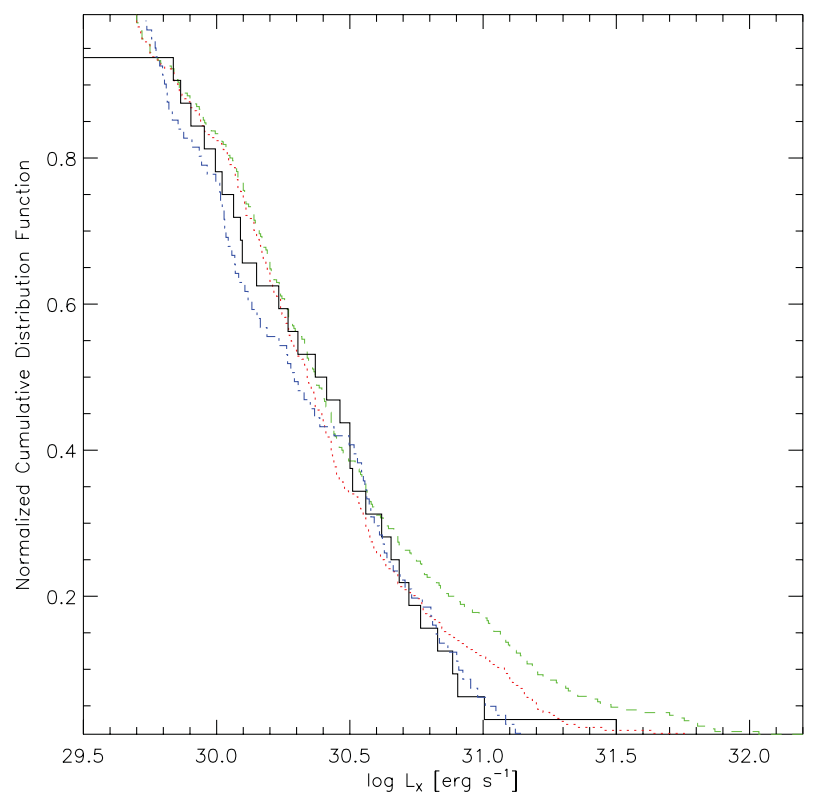

Figure 7. Cumulative distribution function of $\log L_{X}$ in NGC 2023 (continuous line, black), Taurus (dash-dotted line, blue) and in the ONC. The ONC is plotted twice, one for the whole sample (dashed line, green) and the other with stars with masses less than $3 \mathrm{M}_{\odot}$, plotted as a dotted (red) line.

The relationships obtained by us were

$$
\begin{aligned}
f_{\mathrm{X}}[\mathrm{MOS} 1] & =(0.5 \pm 0.3)+(0.056 \pm 0.005) \times \mathrm{CR} \\
f_{\mathrm{X}}[\mathrm{MOS} 2] & =(0.2 \pm 0.4)+(0.057 \pm 0.005) \times \mathrm{CR} \\
f_{\mathrm{X}}[\mathrm{PN}] & =(1.5 \pm 0.2)+(0.003 \pm 0.005) \times \mathrm{CR} .
\end{aligned}
$$

Fluxes determined with these three relationships are compatible. Table A1 gives the flux values determined for our sample from their count rates. We include in that table the 11 stars of Table 3 for completeness. For them, the values of the fluxes are those determined from the spectral fitting. As for the 11 stars for which we could perform spectral fitting (see above), fluxes were transformed into luminosities using the distances obtained from the SED fitting (Table A4).

The comparison of the X-ray luminosity function of the different star-forming regions was made only in the range of completeness of the XMM-Newton observation of NGC $2023\left(\log L_{X}\left[\mathrm{erg} \mathrm{s}^{-1}\right] \geq\right.$ 29.8). Known non-members of the ONC identified by Getman et al. (2005) were discarded for this study. From Fig. 7, NGC 2023 seems to be more similar to the Taurus molecular complex. In both cases, there is a lack of stars brighter than $L_{\mathrm{X}} \sim 10^{31} \mathrm{erg} \mathrm{s}^{-1}$, while the ONC have stars with X-ray luminosities up to $10^{31.5}-10^{32} \mathrm{erg} \mathrm{s}^{-1}$. This result may be explained as differences in the stellar population in each star-forming region. While the ONC have massive stars, they are not present in Taurus and NGC 2023. We have explored this hypothesis by constructing a new COUP sample with stars less massive than $3 \mathrm{M}_{\odot}$ (similar to the mass range in NGC 2023). In Fig. 7, this new sample is plotted as a dotted (red) line. The figure suggests that the COUP sample restricted to the same mass range is more similar to NGC 2023 than the sample without mass restriction. We note here that the ages of the Taurus and ONC samples are not equal and that we could not determine robustly the age of NGC 2023. Therefore, this result must be used with caution. 


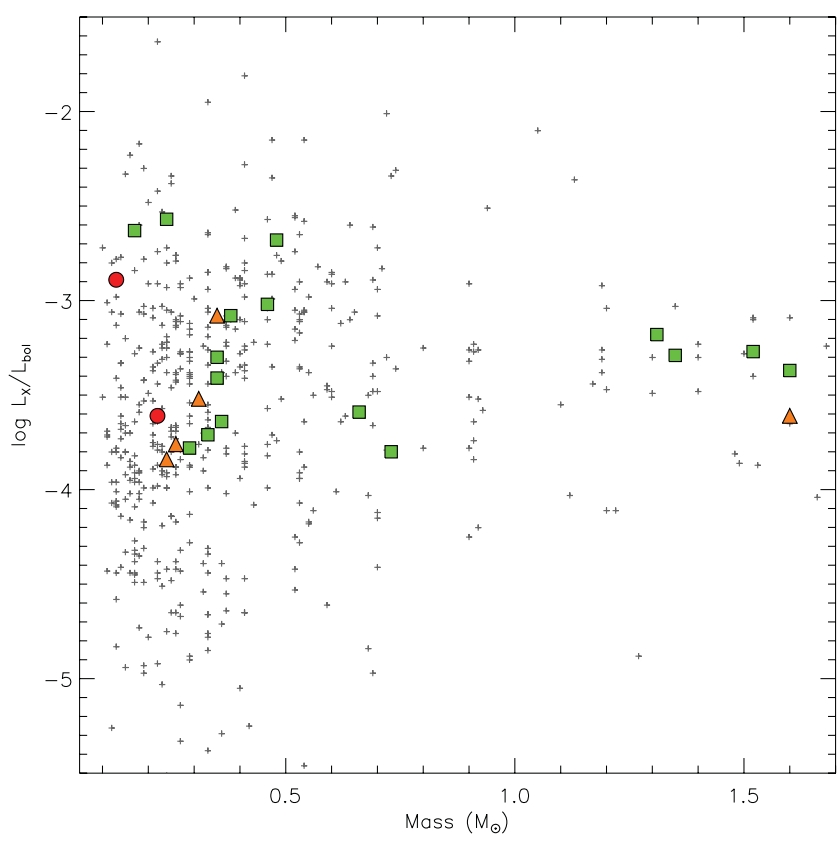

Figure 8. $\log L_{\mathrm{X}} / L_{\mathrm{bol}}$ as a function of stellar mass for the stars of the COUP catalogue (small crosses) and our sample of NGC 2023 stars. Squares are stars classified as class III in Tables A2 and A3. Filled circles are infrared class II objects. Triangles are class II/III objects.

In Fig. 8, we overplot our sample of NGC 2023 sources over the COUP data (Getman et al. 2005). For NGC 2023, we used the masses and bolometric luminosities given in Table A4 and X-ray luminosities determined from the distances given in Table A4 and fluxes of Table A1. All the NGC 2023 low-mass stars $\left(M<1 \mathrm{M}_{\odot}\right)$ are inside the boundaries of the stars of the COUP. Only the more massive stars in our sample (as determined by the SED fitting tool of Robitaille et al. 2007) have very low values of $\log L_{\mathrm{X}} / L_{\mathrm{bol}}$ (out of the limits of Fig. 8, see Table A4). There are three types of sources with very low values of $\log L_{\mathrm{X}} / L_{\mathrm{bol}}$ in our sample: (1) stars showing large infrared excess, for which the SED fitting tool gives very high luminosities, (2) intermediate-mass pre-main-sequence or main-sequence stars (A and F spectral types) and (3) Src 18 that may be an AGN instead of a star (see Section 3.2 and Fig. 5). The SED fitting procedure derives bolometric luminosities and temperatures directly and then determine masses using evolutionary tracks (see Robitaille 2008). For stars with large infrared excesses, to obtain a robust value for their luminosity may be difficult since it depends on the accurate determination of the disc (and envelope in some cases) parameters. Therefore, in those stars of our sample, $L_{\mathrm{bol}}$ may be overestimated.

For the two stars classified as infrared class III objects (i.e. stars without an accretion disc) their temperature may be also overestimated. Nevertheless, the star HD 37805 (Src 3) that is one of these two pre-main-sequence or main-sequence stars with a very low value of the ratio $\log L_{\mathrm{X}} / L_{\mathrm{bol}}$, it is a known A star (e.g. Mookerjea et al. 2009), already detected in X-rays with ASCA (Yamauchi et al. 2000). Therefore, the presence of a low-mass companion responsible for the X-ray emission cannot be discarded in these three intermediate-mass stars in our sample. The other class III object with low $\log L_{\mathrm{X}} / L_{\text {bol }}$ is $\operatorname{Src} 33$ (MIR 83).

\section{SUMMARY AND CONCLUSIONS}

We have carried out a comprehensive analysis of the X-ray and infrared properties of 36 young stellar objects in the NGC 2023 starforming region and its surroundings for the first time. The SED of 90 per cent of this sample (32 out of 36 sources) has been analysed using the star-disc models of Robitaille et al. (2007). We have identified only two AGN candidates among the $36 \mathrm{X}$-ray sources. A classification on different infrared classes has been made according to the position of the stars in infrared colour-colour diagrams and to their SEDs. The study of the SEDs has allowed us to determine several stellar parameters such as masses, radii and luminosities, and other physical parameters such as extinctions and distances.

The X-ray properties of NGC 2023 have been compared with those of other star-forming regions with different characteristics in terms of stellar population. Although the values of temperature and column density determined for our sample of young stars are similar to those observed in stars of the ONC and Taurus, the (X-ray luminosity) cumulative distribution function of members of NGC 2023 is more similar to that of the Taurus molecular complex. A possible explanation is the different stellar populations in each region. While in the ONC there is a number of massive stars, they are not present in Taurus and NGC 2023.

\section{ACKNOWLEDGMENTS}

MAL-G and JL-S acknowledge support by the Spanish Ministerio de Ciencia e Innovación under grant AYA2008-06423-C03-03. JFA$\mathrm{C}$ is a researcher of the CONICET and acknowledges support by grant PICT 2007-02177 (SecyT). PGP-G acknowledges support from the Spanish Programa Nacional de Astronomía y Astrofísica under grants AYA2009-10368 and AYA2009-07723-E. We would like to acknowledge the anonymous referee for a fruitful discussion about the results of this work. His/her comments on the text content have allowed us to notoriously improve this manuscript.

\section{REFERENCES}

Alcalá J. M., Covino E., Torres G., Sterzik M. F., Pfeiffer M. J., Neuhäuser R., 2000, A\&A, 353, 186

Ali B., Sellgren K., Depoy D. L., Carr J. S., Gatley I., Merrill K. M., Lada E., 1998, in Donahue R. A., Bookbinder J. A., eds, ASP Conf. Ser. Vol. 154, Cool Stars, Stellar Systems and the Sun. Astron. Soc. Pac., San Francisco, p. 1663

Anglada G., Rodríguez L. F., 2002, Rev. Mex. Astron. Astrofis., 38, 13

Arnaud K., 1996, in Jacoby G. H., Barnes J., eds, ASP Conf. Ser. Vol. 101, xspec: The First Ten Years. Astron. Soc. Pac., San Francisco, p. 17

Arnaud K., 2004, American Astronomical Society, HEAD meeting 8, 16.29, Bulletin of the American Astronomical Society, Vol. 36, p. 934

Bally J., O'Dell C. R., McCaughrean M. J., 2000, AJ, 119, 2919

Baraffe I., Chabrier G., Allard F., Hauschildt P. H., 1998, A\&A, 337, 403

Barrado D. et al., 2011, A\&A, 526, A21

Bonnarel F. et al., 2000, A\&AS, 143, 33

Bowler B. P., Waller W. H., Megeath S. T., Patten B. M., Tamura M., 2009 , AJ, 137, 3685

Caballero J. A., Solano E., 2008, A\&A, 485, 931

Cutri R. M. et al., 2012, VizieR Online Data Catalog, 2307, 0

DENIS Consortium, 2005, VizieR Online Data Catalog, 2263, 0

Droege T. F., Richmond M. W., Sallman M. P., Creager R. P., 2006, PASP, 118,1666

Droege T. F., Richmond M. W., Sallman M., 2007, VizieR Online Data Catalog, 2271, 0

Eisner J. A., Carpenter J. M., 2003, ApJ, 598, 1341

Fang M., van Boekel R., Wang W., Carmona A., Sicilia-Aguilar A., Henning T., 2009, A\&A, 504, 461 
Genzel R., Stutzki J., 1989, ARA\&A, 27, 41

Getman K. V. et al., 2005, ApJS, 160, 319

Giardino G., Favata F., Micela G., Sciortino S., Winston E., 2007a, A\&A, 463,275

Güdel M. et al., 2007, A\&A, 468, 353

Haisch K. E. Jr, Lada E. A., Lada C. J., 2000, AJ, 120, 1396

Hartmann L., Burkert A., 2007, ApJ, 654, 988

Holland W. S. et al., 1999, MNRAS, 303, 659

Ingleby L. et al., 2009, ApJ, 703, L137

Kaastra J. S., 1992, An X-Ray Spectral Code for Optically Thin Plasmas (Internal SRON-Leiden Report, updated version 2.0)

Kouzuma S., Yamaoka H., 2010, A\&A, 509, A64

Liedahl D. A., Osterheld A. L., Goldstein W. H., 1995, ApJ, 438, L115

López-Santiago J., Caballero J. A., 2008, A\&A, 491, 961

López-Santiago J., Albacete Colombo J. F., López-García M. A., 2010, A\&A, 524, A97

Malin D. F., Ogura K., Walsh J. R., 1987, MNRAS, 227, 361

Mewe R., Gronenschild E. H. B. M., van den Oord G. H. J., 1985, A\&AS, 62,197

Mewe R., Lemen J. R., van den Oord G. H. J., 1986, A\&AS, 65, 511

Meyer M. R., Flaherty K., Levine J. L., Lada E. A., Bowler B. P., Kandori R., 2008, in Reipurth B., ed., Handbook of Star Forming Regions, Vol. I, p. 662

Mookerjea B., Sandell G., Jarrett T. H., McMullin J. P., 2009, A\&A, 507, 1485

Penz T., Micela G., Lammer H., 2007, A\&A, 477, 309

Pérez-González P. G. et al., 2008, ApJ, 675, 234

Pillitteri I., Wolk S. J., Allen L., Megeath S. T., Gutermuth R. A., Gutermuth, 2010a, in Proceedings of the International Astronomical Union, Vol. 5, Symposium S266, August 2009, p. 509

Preibisch T., Feigelson E. D., 2005, ApJS, 160, 390

Preibisch T. et al., 2005, ApJS, 160, 401
Reipurth B., Rodríguez L. F., Anglada G., Bally J., 2004, AJ, 127, 1736

Robitaille T. P., 2008, in ASP Conf. Ser. Vol. 387, Massive Star Formation: Observations Confront Theory. Astron. Soc. Pac., San Francisco, p. 290

Robitaille T. P., Whitney B. A., Indebetouw R., Wood K., 2007, ApJS, 169, 328

Robrade J., Schmitt J. H. M. M., 2007, A\&A, 461, 669

Sandell G. et al., 1999, ApJ, 519, 236

Scally A., Clarke C., 2001, MNRAS, 325, 449

Siess L., Dufour E., Forestini M., 2000, A\&A, 358, 593

Skinner S., Gagné M., Belzer E., 2003, ApJ, 598, 375

Skrutskie M. F. et al., 2006, AJ, 131, 1163

Smith R. K., Brickhouse N. S., Liedahl D. A., Raymond J. C., 2001a, ApJ, 556, L91

Smith R. K., Brickhouse N. S., Liedahl D. A., Raymond J. C., 2001b, ASP Conf. Ser. Vol. 247, Spectroscopic Challenges of Photoionized Plasmas. Astron. Soc. Pac., San Francisco, p. 161

Stahler S. W., Palla F., 2005, The Formation of Stars. Wiley-VCH

Stelzer B., Micela G., Neuhäuser R., 2004, A\&A, 423, 1029

Stelzer B., Preibisch T., Alexander F., Mucciarelli P., Flaccomio E., Micela G., Sciortino S., 2012, A\&A, 537, A135

Stern D. et al., 2005, ApJ, 631, 163

Wilson T. L., Muders D., Dumke M., Henkel C., Kawamura J. H., 2011, ApJ, 728, 61

Wolk S. J., Pillitteri I., Megeath S. T., Allen L. (SOXS Consortium), 2010, BAAS, 42, 683

Yamauchi S., Kamimura R., Koyama K., 2000, PASJ, 52, 1087

\section{APPENDIX A: TABLES AND FIGURES}




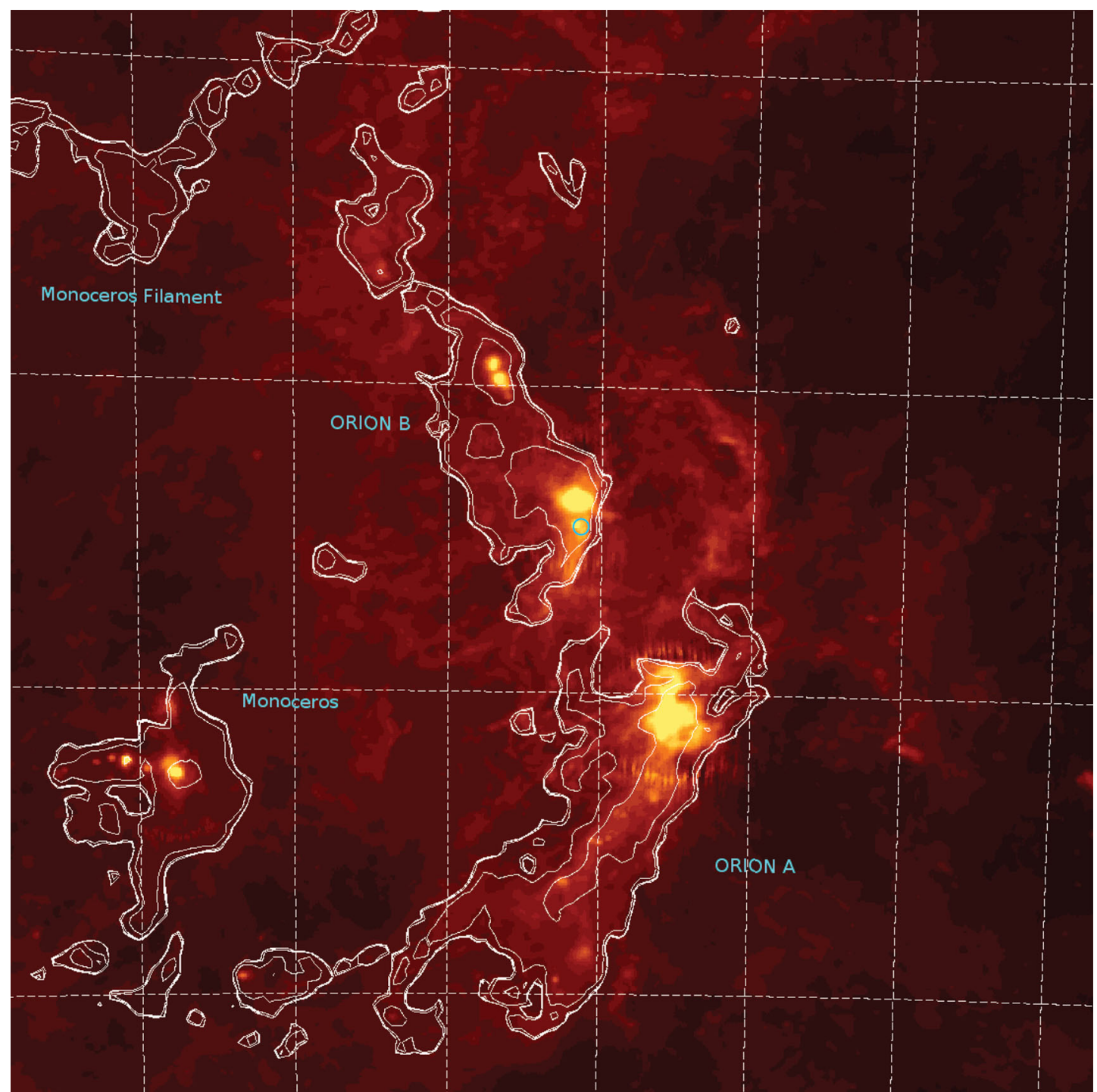

Figure A1. IRAS $100 \mu \mathrm{m}$ mosaic of the complex molecular system Orion A, Orion B and Monoceros. CO map contours are overplotted as continuous lines. The light open circle in the centre of the image represents the position and FoV of the XMM-Newton observation. 


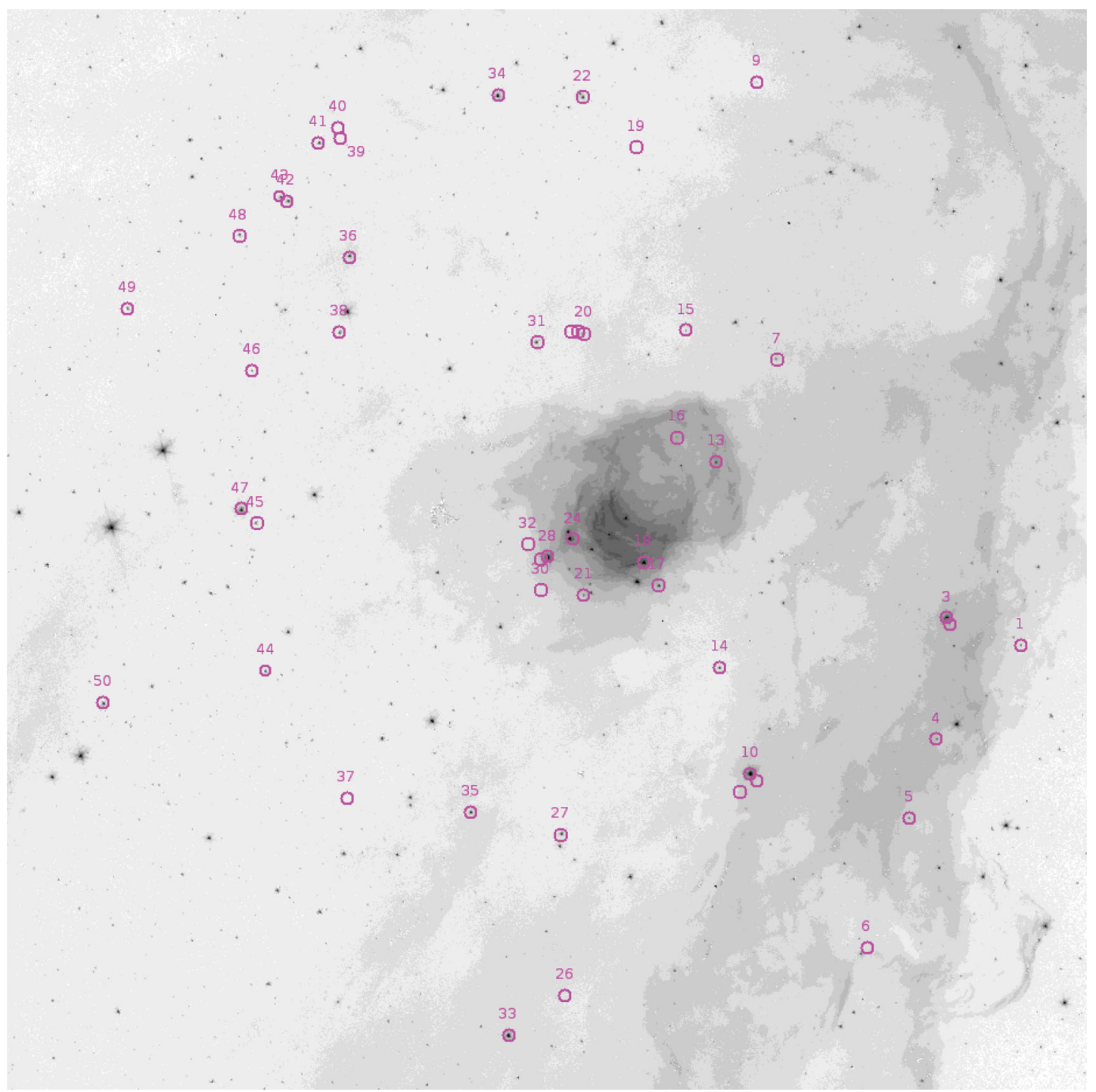

Figure A2. Spitzer-IRAC channel $1(3.6 \mu \mathrm{m})$ image of the NGC 2023 nebula and its surroundings. The Horsehead Nebula is visible at the right bottom corner. $\mathrm{X}$-ray sources detected in the XMM-Newton observation are marked and numbered in magenta. To avoid confusion, the numbers of the likely spurious X-ray sources identified with numbers 11 and 12 (probable multi-detection of Src 10), 23 and 25 (multi-detection of Src 20) and 29 (multi-detection of Src 28) have been removed (see Section 3). 
786

M. A. López-García et al.
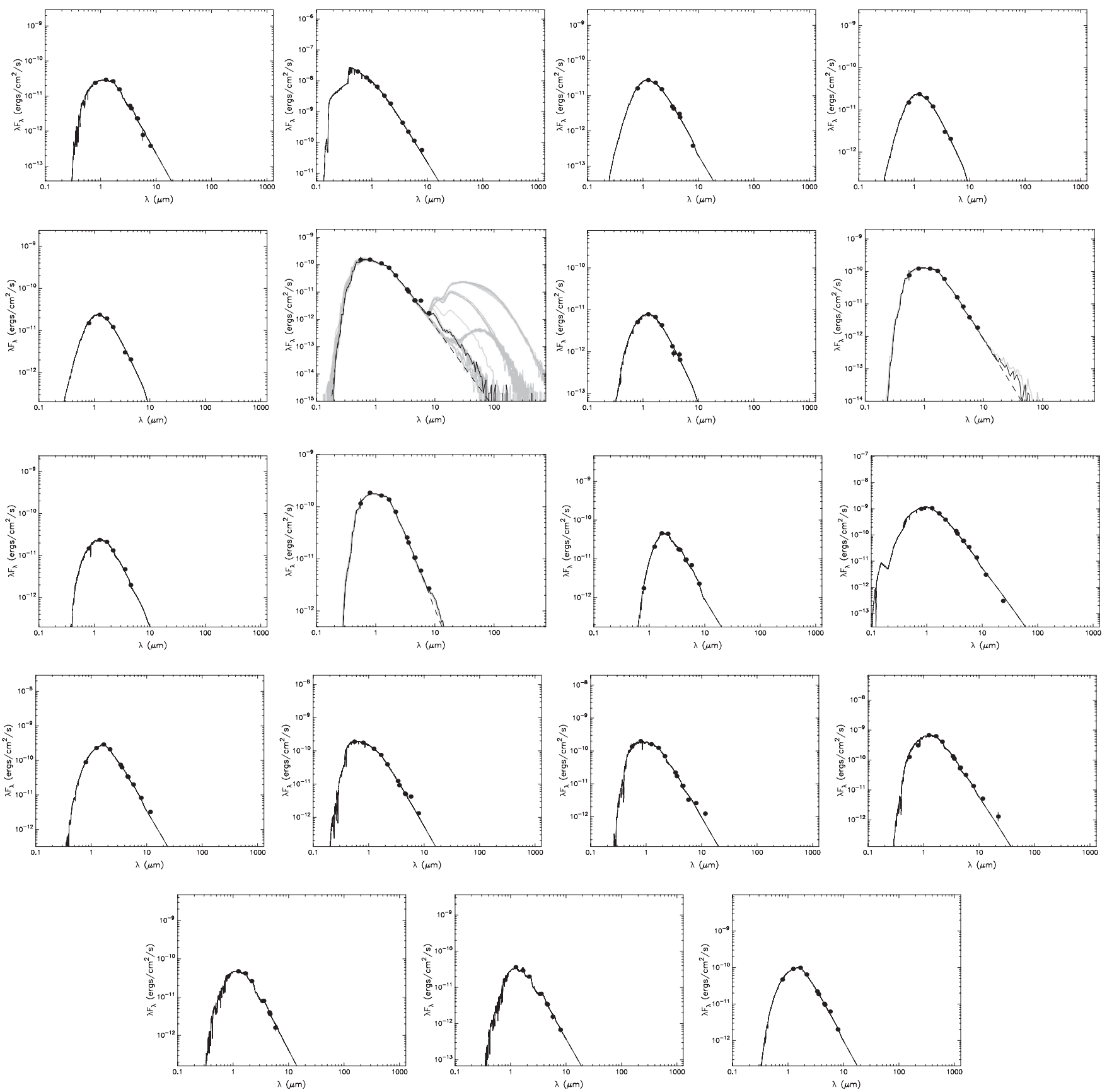

Figure A3. SED of objects classified as class III in Table A3. The solid black lines indicate the best-fitting model using Robitaille et al. (2007). Grey lines are other models with low $\chi^{2}$ values similar to that of the best-fitting model $\left[\left(\chi^{2}-\chi_{\text {best }}^{2}\right) / \chi^{2} \leq 10\right.$ per cent $]$. The dashed lines represent the stellar photosphere for the accepted model. 
NGC 2023 with XMM-Newton and Switzer

787
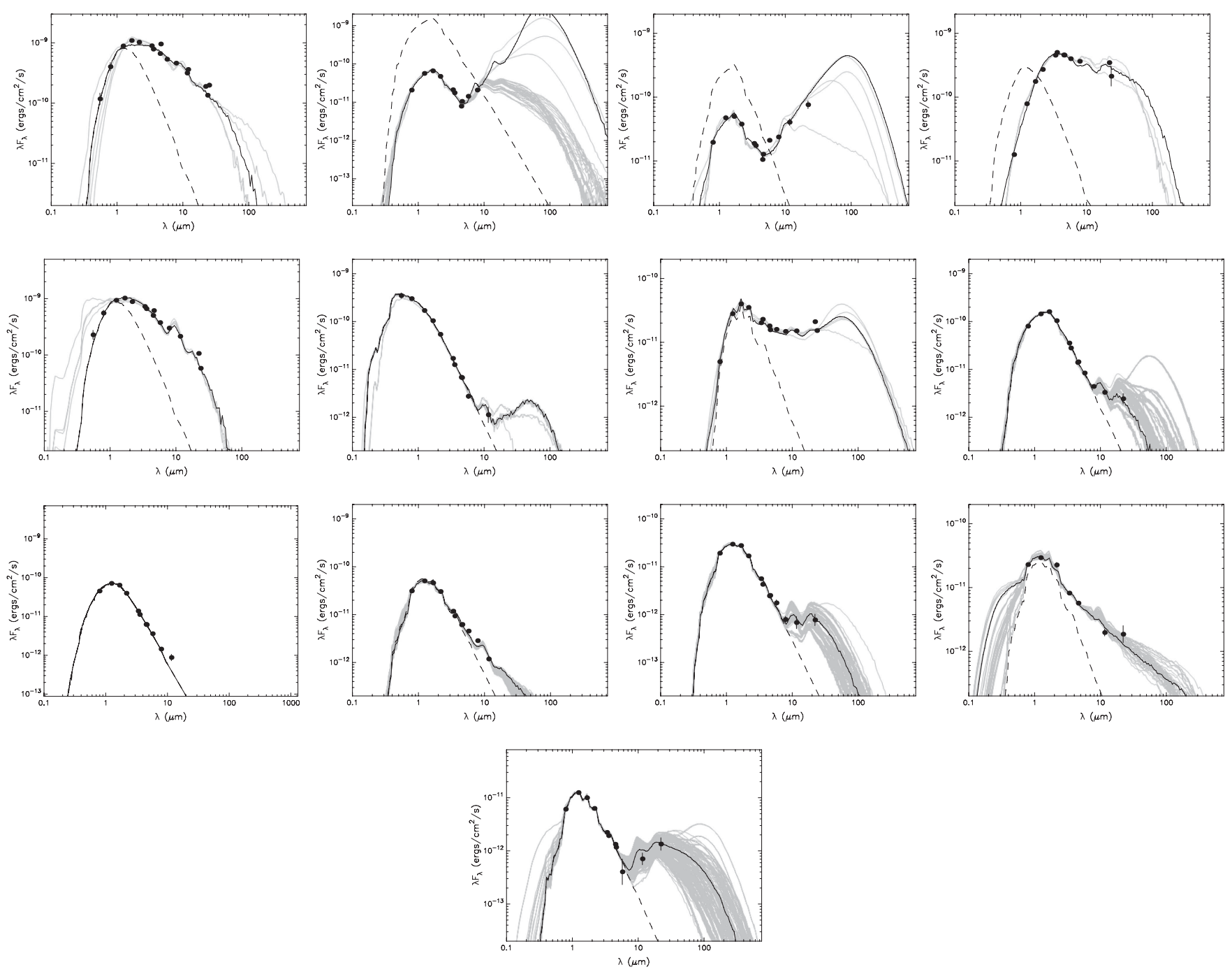

Figure A4. Same as Fig. A3 for stars classified as classes I/II, II and II/III in Table A3. The dashed lines are the unabsorbed stellar REDs. 
Table A1. X-ray parameters from the SAS multitask edetect_chain.

\begin{tabular}{|c|c|c|c|c|c|c|c|c|c|}
\hline Src ID & $\begin{array}{l}\alpha \\
\left({ }^{\circ}\right)\end{array}$ & $\begin{array}{c}\delta \\
\left(^{\circ}\right)\end{array}$ & $\begin{array}{c}\text { Rate [pn] } \\
\left(\mathrm{ks}^{-1}\right)\end{array}$ & $\begin{array}{l}\text { Rate }[\operatorname{mos} 1] \\
\quad\left(\mathrm{ks}^{-1}\right)\end{array}$ & $\begin{array}{l}\text { Rate }[\operatorname{mos} 2] \\
\left(\mathrm{ks}^{-1}\right)\end{array}$ & $\begin{array}{c}\text { Obs. flux } \\
\left(\times 10^{-13} \mathrm{erg} \mathrm{cm}^{-2} \mathrm{~s}^{-1}\right)\end{array}$ & HR1 & $\mathrm{HR} 2$ & Remarks $^{b}$ \\
\hline 1 & 054054.2 & -021904.8 & - & $8 \pm 1$ & $10 \pm 2$ & $0.79 \pm 0.44$ & -0.55 & -0.49 & \\
\hline 4 & 054103.8 & -022139.6 & $12 \pm 2$ & - & $6 \pm 1$ & $0.97 \pm 0.42$ & -0.56 & -0.31 & \\
\hline 5 & 054106.7 & -022352.8 & $13 \pm 2$ & $5 \pm 1$ & $4 \pm 1$ & $0.98 \pm 0.42$ & -0.71 & -1.00 & \\
\hline 6 & 054111.5 & -022725.2 & - & $2 \pm 1$ & $1 \pm 1$ & $0.22 \pm 0.44$ & - & - & \\
\hline 7 & 054121.6 & -021109.6 & $8 \pm 1$ & - & - & $0.93 \pm 0.41$ & -0.53 & -0.15 & [YKK2000] A6 \\
\hline 12 & 054125.7 & -022306.0 & $3 \pm 1$ & - & - & $0.87 \pm 0.41$ & -0.45 & -0.34 & \\
\hline 13 & 054128.3 & -021358.8 & $4 \pm 1$ & - & - & $0.88 \pm 0.41$ & -0.70 & -1.00 & \\
\hline 14 & 054128.3 & -021944.4 & $3 \pm 1$ & - & - & $0.87 \pm 0.41$ & -0.78 & -1.00 & \\
\hline 15 & 054131.9 & -021019.2 & $5 \pm 1$ & - & - & $0.89 \pm 0.41$ & -0.73 & -1.00 & \\
\hline 16 & 054132.6 & -021319.2 & $3 \pm 1$ & - & - & $0.87 \pm 0.41$ & -1.00 & -0.08 & \\
\hline 17 & 054134.8 & -021724.0 & $17 \pm 2$ & $11 \pm 1$ & $9 \pm 1$ & $1.03 \pm 0.15$ & -0.20 & -0.89 & \\
\hline 18 & 054136.5 & -021648.0 & - & $1 \pm 1$ & $1 \pm 1$ & $0.22 \pm 0.44$ & -0.63 & -0.42 & \\
\hline 24 & 054144.2 & -021608.4 & $3 \pm 1$ & - & - & $0.87 \pm 0.41$ & -0.95 & -0.03 & \\
\hline 27 & 054145.6 & -022418.0 & $14 \pm 2$ & $6 \pm 1$ & $5 \pm 1$ & $1.29 \pm 0.38$ & 0.57 & -0.34 & \\
\hline 28 & 054147.0 & -021637.2 & $477 \pm 7$ & $166 \pm 4$ & $159 \pm 4$ & $8.32 \pm 0.87$ & -0.18 & -0.68 & [YKK2000] A11 \\
\hline 31 & 054148.2 & -021040.8 & $7 \pm 1$ & $2 \pm 1$ & - & $0.92 \pm 0.41$ & -0.79 & -0.66 & \\
\hline 33 & 054151.1 & -022952.8 & - & $15 \pm 2$ & $10 \pm 2$ & $0.73 \pm 0.44$ & -0.01 & -0.70 & \\
\hline 34 & 054152.6 & -020350.4 & $131 \pm 6$ & $50 \pm 4$ & $35 \pm 4$ & $4.10 \pm 1.01$ & 0.30 & -0.61 & [YKK2000] A12 \\
\hline 35 & 054155.7 & -022342.0 & $14 \pm 2$ & $3 \pm 1$ & - & $1.26 \pm 0.47$ & 0.16 & -0.77 & \\
\hline 36 & 054209.1 & -020820.4 & $62 \pm 6$ & $16 \pm 2$ & $20 \pm 2$ & $1.48 \pm 0.99$ & -0.05 & -0.55 & \\
\hline 38 & 054210.1 & -021022.8 & $7 \pm 1$ & - & - & $0.92 \pm 0.41$ & -0.91 & -1.00 & \\
\hline 41 & 054212.5 & -020509.6 & $517 \pm 13$ & - & $145 \pm 7$ & $9.90 \pm 0.93$ & -0.36 & -0.53 & \\
\hline 42 & 054216.1 & -020646.8 & $72 \pm 5$ & $27 \pm 3$ & $18 \pm 2$ & $1.57 \pm 0.74$ & -0.71 & -0.61 & [YKK2000] A13 \\
\hline 43 & 054216.8 & -020639.6 & $12 \pm 3$ & $6 \pm 2$ & - & $0.97 \pm 0.42$ & -0.89 & -0.43 & [YKK2000] A13 \\
\hline
\end{tabular}

${ }^{a}$ Flux converted from observed count rates as explained in Section 5. ${ }^{b}$ As given in Tables A2 and A3, we have removed sources considered as spurious; [YKK2000] is for Yamauchi et al. (2000). 
Table A2. Optical and near-infrared magnitudes for the sources detected in the XMM-Newton observation.

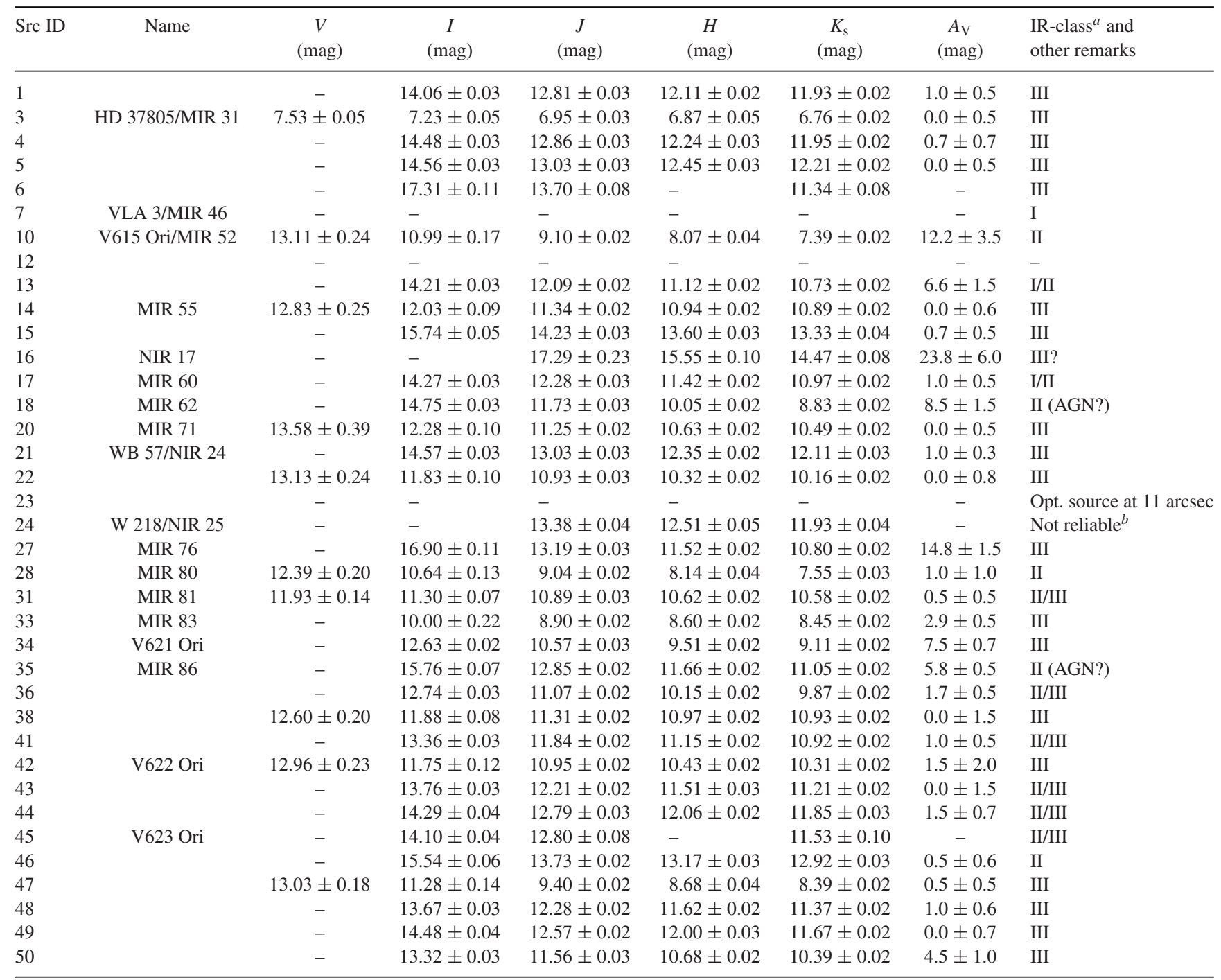

${ }^{a}$ Infrared object class from the SED and Spitzer colour-colour diagrams and remarks for particular sources. We have removed spurious sources (2, 8, 9, 11, $19,25,26,29,30,32,37,39,40) .{ }^{b}$ The photometry of Src 24 is not reliable due to confusion of sources in the region. 


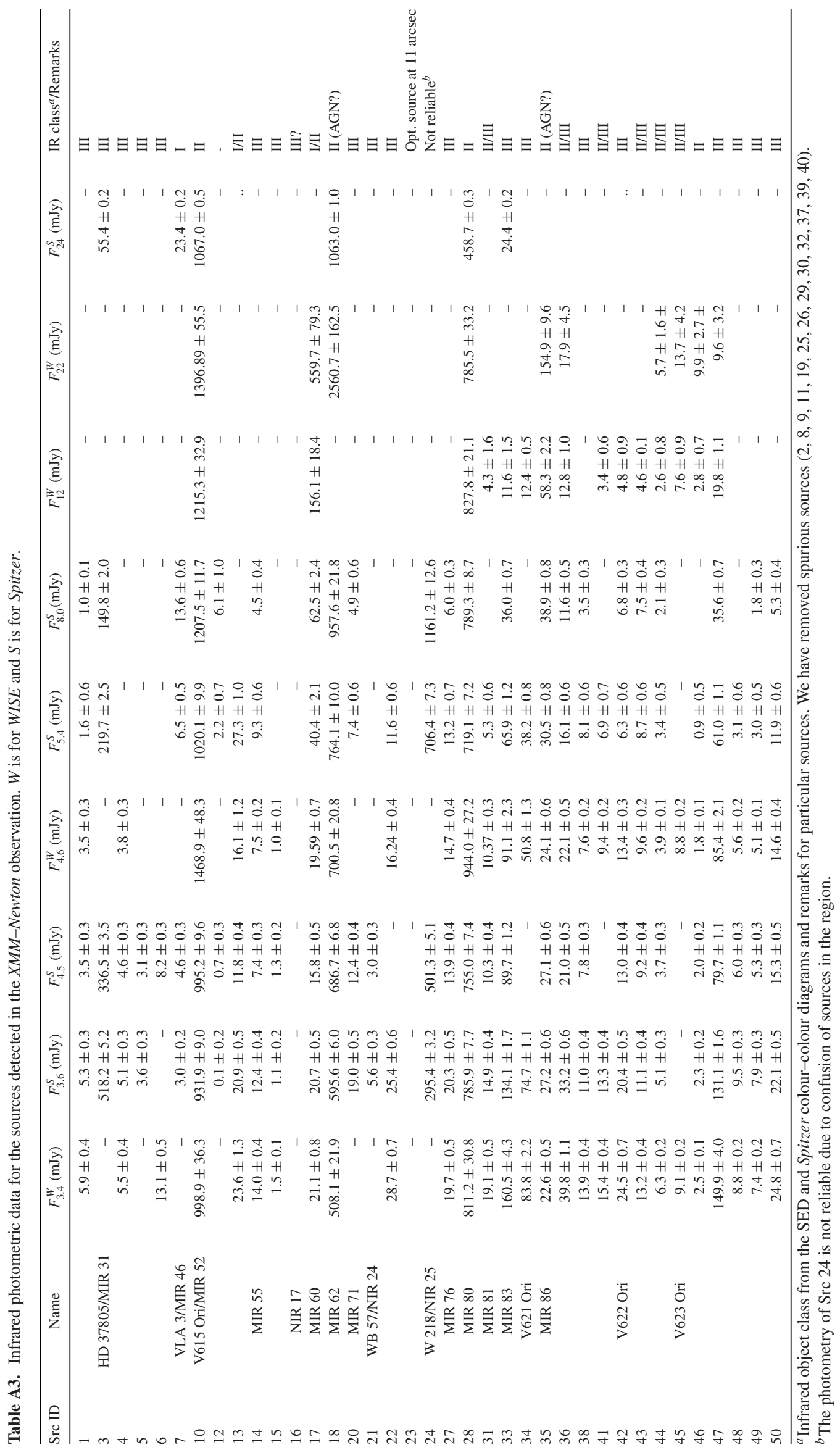


Table A4. Main results from the use of the fitting tool of Robitaille et al. (2007) with the NGC 2023 X-ray detected sources.

\begin{tabular}{|c|c|c|c|c|c|c|c|c|c|c|c|}
\hline Src ID & Name & $\begin{array}{c}A_{\mathrm{V}} \\
(\mathrm{mag})\end{array}$ & $\begin{array}{l}\text { Distance } \\
(\mathrm{pc})\end{array}$ & $\begin{array}{c}M_{\star} \\
\left(\mathrm{M}_{\odot}\right)\end{array}$ & $\begin{array}{c}R_{\star} \\
\left(\mathrm{R}_{\odot}\right)\end{array}$ & $\begin{array}{c}T_{\star} \\
(\mathrm{K})\end{array}$ & $\begin{array}{c}L_{\text {bol }} \\
\left(\mathrm{L}_{\odot}\right)\end{array}$ & $\log L_{\mathrm{X}} / L_{\mathrm{bol}}$ & $\begin{array}{c}\dot{M} \\
\left(\mathrm{M}_{\odot} \mathrm{yr}^{-1}\right)\end{array}$ & Stage $^{a}$ & IR class ${ }^{b}$ \\
\hline 1 & & 0.05 & 398 & 0.48 & 1.02 & 3735 & 0.19 & -2.68 & - & - & III \\
\hline 3 & HD 37805/MIR 31 & 0.10 & 56 & 1.45 & 1.77 & 7500 & 8.53 & -13.02 & - & - & III \\
\hline 4 & & 1.11 & 299 & 0.35 & 0.77 & 3550 & 0.09 & -3.41 & - & - & III \\
\hline 5 & & 0.25 & 398 & 0.38 & 0.52 & 3650 & 0.01 & -3.08 & - & - & III \\
\hline 6 & & 8.81 & 630 & 0.66 & 0.76 & 4672 & 0.27 & -3.59 & - & - & III \\
\hline 10 & V615 Ori/ MIR 52 & 5.19 & 371 & 2.96 & 4.39 & 7902 & 67.4 & -69.89 & $1.82 \times 10^{-9}$ & III & II \\
\hline 13 & & 1.90 & 500 & 1.09 & 10.14 & 4037 & 24.6 & -27.52 & $1.03 \times 10^{-8}$ & I & $\mathrm{I} / \mathrm{II}$ \\
\hline 14 & MIR 55 & 0.00 & 575 & 1.60 & 1.88 & 5084 & 2.11 & -3.37 & - & - & III \\
\hline 15 & & 0.24 & 479 & 0.29 & 0.76 & 3399 & 0.07 & -3.78 & - & - & III \\
\hline 17 & MIR 60 & 1.19 & 575 & 0.48 & 5.40 & 3692 & 4.87 & -8.36 & $2.15 \times 10^{-9}$ & I & $\mathrm{I} / \mathrm{II}$ \\
\hline 18 & MIR 62 & 5.33 & 457 & 3.42 & 2.63 & 11725 & 118 & -121.6 & $4.51 \times 10^{-9}$ & III & II \\
\hline 20 & MIR 71 & 0.76 & 347 & 1.31 & 1.46 & 4809 & 1.02 & -3.18 & - & - & III \\
\hline 21 & NIR 24 & 0.42 & 245 & 0.24 & 0.72 & 3366 & 0.06 & -2.57 & - & - & III \\
\hline 22 & & 0.34 & 288 & 1.35 & 1.52 & 4886 & 1.18 & -3.29 & - & - & III \\
\hline 27 & MIR 76 & 7.65 & 275 & 0.35 & 1.92 & 3500 & 0.63 & -3.30 & - & - & III \\
\hline 28 & MIR 80 & 3.11 & 436 & 2.96 & 4.39 & 7902 & 67.4 & -69.09 & $1.82 \times 10^{-9}$ & III & II \\
\hline 31 & MIR 81 & 0.88 & 616 & 1.85 & 1.53 & 7978 & 9.32 & -12.03 & $1.70 \times 10^{-14}$ & III & II/III \\
\hline 33 & MIR 83 & 2.15 & 280 & 1.93 & 2.78 & 6835 & 15.1 & -4.92 & - & - & III \\
\hline 34 & V621 Ori & 4.30 & 309 & 2.05 & 2.96 & 4828 & 4.27 & -3.54 & - & - & III \\
\hline 35 & MIR 86 & 3.87 & 550 & 0.22 & 2.86 & 3119 & 0.93 & -3.61 & $1.07 \times 10^{-7}$ & I & II \\
\hline 36 & & 2.88 & 309 & 1.60 & 1.91 & 4847 & 1.82 & -3.61 & $2.70 \times 10^{-13}$ & III & II/III \\
\hline 38 & & 0.07 & 199 & 0.33 & 0.47 & 3500 & 0.01 & -3.71 & - & - & III \\
\hline 41 & & 0.55 & 166 & 0.35 & 0.77 & 3567 & 0.09 & -3.08 & $2.54 \times 10^{-10}$ & III & II/III \\
\hline 42 & V622 Ori & 0.11 & 407 & 1.52 & 1.83 & 4759 & 1.54 & -3.27 & $1.13 \times 10^{-13}$ & III & III \\
\hline 43 & & 0.37 & 182 & 0.24 & 0.82 & 3343 & 0.08 & -3.84 & $1.53 \times 10^{-11}$ & III & II/III \\
\hline 44 & & 0.51 & 257 & 0.31 & 0.29 & 3556 & 0.02 & -3.52 & $7.88 \times 10^{-12}$ & III & II/III \\
\hline 45 & V623 Ori & 0.13 & 309 & 0.26 & 0.27 & 3401 & 0.01 & -3.76 & $1.09 \times 10^{-8}$ & III & II/III \\
\hline 46 & & 0.07 & 500 & 0.13 & 0.15 & 3052 & 0.002 & -2.89 & $3.07 \times 10^{-11}$ & III & II \\
\hline 47 & & 1.49 & 148 & 0.73 & 0.61 & 4331 & 0.13 & -3.80 & $3.00 \times 10^{-14}$ & III & III \\
\hline 48 & & 0.09 & 275 & 0.36 & 1.00 & 3548 & 1.43 & -3.64 & - & - & III \\
\hline 49 & & 0.29 & 398 & 0.17 & 0.16 & 3042 & 0.20 & -2.63 & - & - & III \\
\hline 50 & & 1.37 & 190 & 0.46 & 1.11 & 3706 & 0.21 & -3.02 & - & - & III \\
\hline
\end{tabular}

${ }^{a}$ Evolutionary stage parameter from Robitaille et al. (2007). ${ }^{b}$ Infrared class based on the SEDs (Tables A2 and A3).

This paper has been typeset from a $\mathrm{T}_{\mathrm{E}} \mathrm{X} / \mathrm{LT} \mathrm{E} \mathrm{X}$ file prepared by the author. 